\title{
A characteristic initial boundary value problem for a symmetric positive system
}

\author{
(Dedicated to Professor Kôji Kubota on his 60th birthday)
}

\section{Tatsuo Nishitani and Masahiro TAKAYAMA}

(Received May 22, 1995)

\begin{abstract}
We study the simplest maximal positive boundary value problem for symmetric positive systems in a bounded open set for which the boundary matrix is not of constant rank. To be precise, the boundary matrix changes the definiteness simply crossing an embedded manifold in the boundary which is the intersection of the boundary with a non-characteristic hypersurface. Assuming that the flow passing the hypersurface compensates for the degeneracy of the boundary matrix on the embedded manifold, we discuss the existence of regular solutions to the boundary value problem.
\end{abstract}

Key words: Symmetric positive boundary value problem, characteristic boundary, not of constant rank, maximal positive boundary condition.

\section{Introduction}

Let $\Omega$ be a bounded open subset of $\mathbf{R}^{n}$ with smooth boundary $\partial \Omega$. In $\Omega$, we consider a first order symmetric system

$$
\begin{aligned}
& L u=\sum_{j=1}^{n} A_{j}(x) \partial_{j} u+B(x) u, \\
& A_{j}(x), B(x) \in C^{\infty}(\bar{\Omega}), \quad A_{j}^{*}(x)=A_{j}(x)
\end{aligned}
$$

with $u=\left(u_{1}, \ldots, u_{N}\right)$ and $\partial_{j}=\partial / \partial x_{j}$. For $x \in \partial \Omega$ we denote by

$$
A_{b}(x)=\sum_{j=1}^{n} \nu_{j} A_{j}(x)
$$

the boundary matrix where $\nu=\left(\nu_{1}, \ldots, \nu_{n}\right)$ is the unit outward normal to $\Omega$.

In this paper we concerned with the case that $A_{b}(x)$ changes the definiteness simply crossing an embedded $n-2$ dimensional submanifold $\gamma$ of $\partial \Omega$ defined as $\gamma=\partial \Omega \cap\{h(x)=0\}$ where $h(x)$ is a smooth function in a neighborhood of $\partial \Omega$. Let us make our assumptions precise. Take $r(x) \in C^{\infty}\left(\mathbf{R}^{n}\right)$ 
with $d r(x) \neq 0$ on $\partial \Omega$ so that $\Omega=\{r(x)>0\}$ and let

$$
A_{r}(x)=\sum_{j=1}^{n}\left(\partial_{j} r\right)(x) A_{j}(x) .
$$

Our first assumption of this paper is stated as

$$
A_{r}(x)=-h(x) A(x) \quad \text { in } \quad V \cap \Omega
$$

with a smooth definite $A(x)$ where $V$ is a neighborhood of $\partial \Omega$.

Since $A_{b}(x)=-A_{r}(x) /|d r(x)|$ on $\partial \Omega$, it is clear that $A_{b}(x)$ has the same definiteness as $A(x)$ on $\Gamma^{+}=\partial \Omega \cap\{h(x)>0\}$ and the opposite definiteness on $\Gamma^{-}=\partial \Omega \cap\{h(x)<0\}$.

The boundary condition takes the form

$$
u(x) \in M(x) \quad \text { for } \quad x \in \partial \Omega
$$

where $M(x)(x \in \partial \Omega)$ is a linear subspace of $\mathbf{C}^{N}$. We suppose that $M$ is maximal positive in the sense that

$$
\begin{gathered}
\left\langle A_{b}(x) v, v\right\rangle \geq 0, \quad \forall x \in \partial \Omega, \quad \forall v \in M(x), \\
\operatorname{dim} M(x)=\#\left\{\text { nonnegative eigenvalues of } A_{b}(x)\right. \\
\text { counting multiplicity }\}
\end{gathered}
$$

Then (1.1) and the maximality condition imply that

$$
M(x)= \begin{cases}\mathbf{C}^{N} & \text { if } \quad x \in \Gamma^{+} \cup \gamma \quad\left(\text { resp. } \Gamma^{-} \cup \gamma\right) \\ \{0\} & \text { if } \quad x \in \Gamma^{-} \quad\left(\text { resp. } \Gamma^{+}\right)\end{cases}
$$

if $A(x)$ (resp. $-A(x))$ is positive definite.

We study the following boundary value problem

$(\mathrm{BVP})$

$$
\left\{\begin{array}{rll}
(L+\lambda) u=f & \text { in } & \Omega \\
u \in M & \text { at } & \partial \Omega .
\end{array}\right.
$$

When $\operatorname{dimKer} A_{b}(x)$ is constant on $\partial \Omega$, in Rauch [8], we can find detailed studies of boundary value problems for symmetric positive boundary conditions, including refined and simplified standard classical results ([2], [4]). See also the references given there.

When $\operatorname{dimKer} A_{b}(x)$ is not constant on $\partial \Omega$, Rauch [9] studied the $L^{2}$ structure of the problem (BVP) and proved that weak is strong assuming that, locally, in each component of $\partial \Omega \backslash \gamma$ the boundary space $M(x)$ coincides 
with a smooth linear subspace $N_{\text {small }}(x)$ or $N_{\text {big }}(x)$ (of fixed dimensions) with $N_{\text {small }}(x) \subset N_{\text {big }}(x)$ and $\operatorname{Ker} A_{b}(x) \subset N_{\text {big }}(x)$ (see also [5], [6], [7]).

Since our case is a special one studied in $[9]$ it follows that weak solutions are strong. However our main concern is $H_{s}$ regularity of solutions $u$ to (BVP) with smooth $f$, which serves to study non linear perturbations. As is easily seen, strong solutions $u$ to (BVP) need not be regular for smooth $f$ even in our simple case (see Example 2.1 below). Hence, to get regularity results, we introduce another condition. Let us set

$$
A_{h}(x)=\sum_{j=1}^{n}\left(\partial_{j} h\right)(x) A_{j}(x)
$$

Then our second assumption is:

$$
A_{h}(x) \text { has the same definiteness as } A(x) \text { on } \gamma \text {. }
$$

To visualize the meaning of this condition see Example 2.1 below.

Under the conditions (1.1) and (1.2) we discuss the existence of regular solutions to (BVP). Main results are described in section 2 and proved in section 4 . Section 3 is devoted to some preliminaries. In section 5 we discuss the initial boundary value problem

(IBVP)

$$
\left\{\begin{aligned}
\partial_{t} u+L u=f(t, x) & \text { in } \quad(0, \infty) \times \Omega \\
u(0, x)=u_{0}(x) & \text { in } \Omega \\
u(t, x) \in M(x) & \text { for } \quad(t, x) \in[0, \infty) \times \partial \Omega
\end{aligned}\right.
$$

and prove the existence of regular solutions to (IBVP) assuming (1.1) and (1.2) again (Theorem 5.5).

The authors thank A. Matsumura for directing thier attentions to this problem and for several stimulating discussions related to this subject.

\section{Main results}

To fix the idea, in what follows, we assume that

$$
A(x) \text { is positive definite. }
$$

Otherwise it is enough to take $-h(x)$ for $h(x)$. We denote the formal adjoint of $L$ by $L^{*}$ 


$$
L^{*} u=-\sum_{j=1}^{n} \partial_{j} A_{j}(x) u+B^{*}(x) u .
$$

For $u, v \in C^{1}(\bar{\Omega})$, Green's identity yields

$$
((L+\lambda) u, v)_{L^{2}(\Omega)}=\left(u,\left(L^{*}+\bar{\lambda}\right) v\right)_{L^{2}(\Omega)}+\int_{\partial \Omega}\left\langle A_{b} u, v\right\rangle d \sigma .
$$

The adjoint boundary space $M^{*}(x)$ is defined by

$$
M^{*}(x)=\left[A_{b}(x) M(x)\right]^{\perp} .
$$

From (1.1) it is clear that

$$
M^{*}(x)=\left\{\begin{array}{lll}
\{0\} & \text { if } & x \in \Gamma^{+} \\
\mathbf{C}^{N} & \text { if } & x \in \Gamma^{-} \cup \gamma .
\end{array}\right.
$$

We recall the following definition (see [1], [2]).

Definition For $f \in L^{2}(\Omega), u \in L^{2}(\Omega)$ is a weak solution to $(L+\lambda) u=f$ in $\Omega, u \in M$ at $\partial \Omega$ if and only if the identity

$$
\left(u,\left(L^{*}+\bar{\lambda}\right) \psi\right)_{L^{2}(\Omega)}=(f, \psi)_{L^{2}(\Omega)}
$$

holds for all $\psi \in C^{\infty}(\bar{\Omega})$ with $\psi \in M^{*}$ at $\partial \Omega$.

We now introduce some function spaces. By $D_{\infty}\left(\Omega, \Gamma^{-}\right)$we denote the set of all functions $u \in C^{\infty}(\bar{\Omega})$ satisfying supp $u \cap\left(\Gamma^{-} \cup \gamma\right)=\emptyset$. By $X_{r}\left(\Omega, \Gamma^{-}\right), r \in \mathbf{Z}_{+}$, we denote the completion of $D_{\infty}\left(\Omega, \Gamma^{-}\right)$under the $H_{r}(\Omega)$ norm where $H_{r}(\Omega)$ is the usual Sobolev space of order $r$. The space $D_{\infty}\left(\Omega, \Gamma^{+}\right)$and $X_{r}\left(\Omega, \Gamma^{+}\right)$are defined similarly. It is clear that

$$
\dot{H}_{r}(\Omega) \subset X_{r}\left(\Omega, \Gamma^{ \pm}\right) \subset H_{r}(\Omega)
$$

for $r \in \mathbf{Z}_{+}$where $\dot{H}_{r}(\Omega)$ is the completion of $C_{0}^{\infty}(\Omega)$ under the $H_{r}(\Omega)$ norm. We now have

Theorem 2.1 For $r \in \mathbf{Z}_{+}$there is a $\Lambda(r) \in \mathbf{R}$ verifying the following properties: Let $u \in L^{2}(\Omega), f \in X_{r}\left(\Omega, \Gamma^{-}\right), \operatorname{Re} \lambda>\Lambda(r)$ and assume that $u$ is a weak solution to $(L+\lambda) u=f$ in $\Omega, u \in M$ at $\partial \Omega$. Then it follows that $u \in X_{r}\left(\Omega, \Gamma^{-}\right)$and

$$
(\operatorname{Re} \lambda-\Lambda(r))\|u\|_{H_{r}(\Omega)} \leq\|(L+\lambda) u\|_{H_{r}(\Omega)} .
$$


This theorem is an immediate consequence of the following two results.

Theorem 2.2 For $r \in \mathbf{Z}_{+}$there is a $\Lambda(r) \in \mathbf{R}$ verifying the following properties: Let $f \in X_{r}\left(\Omega, \Gamma^{-}\right)$and $\operatorname{Re} \lambda>\Lambda(r)$. Then there exists a solution $u \in X_{r}\left(\Omega, \Gamma^{-}\right)$to (BVP) satisfying

$$
(\operatorname{Re} \lambda-\Lambda(r))\|u\|_{H_{r}(\Omega)} \leq\|f\|_{H_{r}(\Omega)} .
$$

If $r \geq 1$, we can really take the trace of $u$ to the boundary so that the trace verifies the boundary condition.

Proposition 2.3 There is a $\Lambda \in \mathbf{R}$ verifying the following properties: Let $f \in L^{2}(\Omega)$ and $\operatorname{Re} \lambda>\Lambda$. Then weak solution $u \in L^{2}(\Omega)$ to (BVP) is unique.

To get regularity results, the assumption (1.2) could not be dropped in general. Indeed we have

Example 2.1. We work in $\mathbf{R}^{2}$. Let $r(x)=\left(4-|x|^{2}\right)\left(|x|^{2}-1\right)$ and let $h(x) \in C^{\infty}\left(\mathbf{R}^{2}\right)$ be so that

$$
h(x)=-x_{1} \quad \text { if } \quad|x|<4 / 3, \quad h(x)=x_{1} \quad \text { if } \quad|x|>5 / 3 .
$$

Recall that $\Omega=\{1<|x|<2\}, \gamma=\{(0, \pm 1),(0, \pm 2)\}$ and $\Gamma^{-}=\{|x|=$ $\left.1, x_{1}>0\right\} \cup\left\{|x|=2, x_{1}<0\right\}$. Let us consider $L=\partial_{1}$. Since $A_{r}=$ $-2 x_{1}\left(|x|^{2}-1\right)+2 x_{1}\left(4-|x|^{2}\right),(1.1)$ is fulfilled. But since $A_{h}=-1$ at $(0, \pm 1)$ and $A_{h}=1$ at $(0, \pm 2),(1.2)$ is not. We now take $g \in C_{0}^{\infty}(\Omega)$ so that

$$
g(x) \geq 0, g(x) \not \equiv 0 \quad \text { if } \quad x_{1} \leq 0, x_{2}=1
$$

and define $v(x)$ in $\Omega$ as

$$
v(x)= \begin{cases}\int_{0}^{x_{1}} g\left(s, x_{2}\right) d s & \text { if } x_{1}>0,\left|x_{2}\right|<1 \\ \int_{-\infty}^{x_{1}} g\left(s, x_{2}\right) d s & \text { otherwise. }\end{cases}
$$

Let $\lambda \in \mathbf{C}$ and set $u(x)=e^{-\lambda x_{1}} v(x)$ and $f(x)=e^{-\lambda x_{1}} g(x)$. Then it is easy to see that $f \in C_{0}^{\infty}(\Omega),(L+\lambda) u=f$ in $\Omega$ and $u=0$ on $\Gamma^{-}$, so that $u$ is a weak solution to (BVP). On the other hand, working near $(0,1)$, it is clear that $u \notin H_{1}(\Omega)$.

We make a comment on the space $X_{r}\left(\Omega, \Gamma^{-}\right)$. To obtain regularity results we could not replace $X_{r}\left(\Omega, \Gamma^{-}\right)$by $H_{r}(\Omega)$ in Theorem 2.1 in general. 
Example 2.2. As in Example 2.1 we work in $\mathbf{R}^{2}$. Let $r(x)=1-|x|^{2}$ and $h(x)=x_{1}$ so that $\Omega=\{|x|<1\}, \gamma=\{(0, \pm 1)\}$ and $\Gamma^{-}=\left\{|x|=1, x_{1}<0\right\}$. Take $L=\partial_{1}$. Since $A_{r}=-2 x_{1}$ and $A_{h}=1$ then (1.1) and (1.2) are fulfilled. Let $\lambda \in \mathbf{C}$ and set $\phi(x)=\left(1-x_{2}^{2}\right)^{1 / 2}+x_{1}, u(x)=e^{-\lambda x_{1}} \phi(x)$ and $f(x)=e^{-\lambda x_{1}}$. Noting that $L \phi=1$ in $\Omega$ and $\phi=0$ on $\Gamma^{-}$it is clear that $u$ is a weak solution to (BVP). On the other hand, we have $u \notin H_{2}(\Omega)$ in spite of $f \in C^{\infty}(\bar{\Omega})$.

\section{Preliminaries}

In this section we introduce a weight function $\phi(x)$ which plays a crucial role in proving the existence of smooth solutions to (BVP). Let

$$
m(x)=\left(h(x)^{2}+2 \mu r(x)\right)^{1 / 2}, \quad \phi(x)=m(x)+h(x)
$$

where $\mu$ is a small positive constant which will be determined in Lemma 3.4 below. Note that $\phi=0$ on $\Gamma^{-}$and $\phi>0$ on $\Gamma^{+} \cup \Omega$. Let us set

$$
\begin{aligned}
& \phi_{\eta}(x)=\phi(x)-\eta, \quad \Omega_{\eta}=\Omega \cap\left\{\phi_{\eta}>0\right\} \\
& L_{(\eta, s)}=\phi_{\eta}^{-s} L \phi_{\eta}^{s}, \quad L_{(\eta, s)}^{*}=\phi_{\eta}^{s} L^{*} \phi_{\eta}^{-s}
\end{aligned}
$$

for $\eta \geq 0$ and $s \in \mathbf{R}$. Note that $\Omega \cap\left\{\phi_{\eta}=0\right\}$ is a union of smooth surfaces if $\eta>0$ is small enough.

We write $(\cdot, \cdot)_{\Omega_{\eta}}$ and $\|\cdot\|_{\Omega_{\eta}}$ for the inner product and the norm in $L^{2}\left(\Omega_{\eta}\right)$ respectively. By $D_{r}\left(\Omega, \Gamma^{-}\right), r \in \mathbf{Z}_{+}$, we denote the set of all functions $u \in$ $H_{r}(\Omega)$ with supp $u \cap\left(\Gamma^{-} \cup \gamma\right)=\emptyset$. The spaces $D_{r}\left(\Omega, \Gamma^{+}\right)$are defined similarly. The following two lemmas are easily checked by standard arguments.

Lemma 3.1 If $u \in D_{0}\left(\Omega, \Gamma^{-}\right)$and $(L+\lambda) u \in L^{2}(\Omega)$, then we can choose a sequence $\left\{u_{\epsilon}\right\} \subset D_{\infty}\left(\Omega, \Gamma^{-}\right)$such that $u_{\epsilon} \rightarrow u,(L+\lambda) u_{\epsilon} \rightarrow(L+\lambda) u$ in $L^{2}(\Omega)$.

Lemma 3.2 Let $u \in D_{0}\left(\Omega, \Gamma^{-}\right), f \in L^{2}(\Omega)$ and assume that $(L+\lambda) u=f$ in $\Omega$. Then $u$ is a weak solution to (BVP).

We now set

$$
G(x)=\sum_{j=1}^{n}\left(\partial_{j} \phi\right)(x) A_{j}(x) .
$$

Then we have 
Lemma 3.3 Let $G(x)$ be as above. Then

(i) $G(x)=m(x)^{-1}\left(\mu A_{r}(x)+\phi(x) A_{h}(x)\right)$. In particular,

$$
G(x)=m(x)^{-1}\left(-\mu h(x) A(x)+\phi(x) A_{h}(x)\right) \quad \text { in } \quad V \cap \Omega .
$$

(ii) $G(x)$ is bounded in $\Omega$.

(iii) $L_{(\eta, s)}=L+s \phi_{\eta}^{-1} G$.

Proof. Since $\partial_{j} \phi=m^{-1}\left(\mu \partial_{j} r+\phi \partial_{j} h\right)$, the first assertion of (i) is clear. The second assertion of (i) follows from (1.1). Since $\phi / m$ and $h / m$ are bounded in $V \cap \Omega, G$ is also bounded there. The assertion (iii) is clear.

Our assumptions imply that $G(x)$ is positive definite near $\Gamma^{-}$. Indeed Lemma 3.4 We can choose $\mu>0$ and a neighborhood $W$ of $\Gamma^{-} \cup \gamma$ so that

$$
G(x) \gg \delta I \text { in } W \cap \Omega
$$

with some $\delta>0$.

Proof. By (1.2) we can choose a small neighborhood $W_{1} \subset V$ of $\gamma$ such that $A_{h}(x)$ is positive definite in $\overline{W_{1} \cap \Omega}$. Then we can find $\mu>0$ so that

$$
\delta_{1} A_{h}(x) \ll \mu A(x) \ll \delta_{2} A_{h}(x) \quad \text { in } \quad W_{1} \cap \Omega
$$

with some $0<\delta_{1}<\delta_{2}<1$. If $x \in W_{1} \cap \Omega$ and $h(x) \geq 0$, then we have

$$
G(x) \gg m^{-1}\left(-\delta_{2} h+\phi\right) A_{h} \gg A_{h} \gg \delta I
$$

with some $\delta>0$. On the other hand, if $x \in W_{1} \cap \Omega$ and $h(x)<0$, then we have

$$
G(x) \gg m^{-1}\left(-\delta_{1} h+\phi\right) A_{h} \gg \delta_{1} A_{h} \gg \delta I .
$$

This proves that $G(x) \gg \delta I$ in $W_{1} \cap \Omega$.

Since $A_{r}$ is positive definite on $\Gamma^{-}$and $\phi=0$ on $\Gamma^{-}$, we can choose a neighborhood $W_{2}$ of $\Gamma^{-} \backslash W_{1}$ so that $G(x) \gg \delta I$ in $\overline{W_{2} \cap \Omega}$. Thus $W=$ $W_{1} \cup W_{2}$ is a desired neighborhood of $\Gamma^{-} \cup \gamma$. 


\section{Proof of main results}

To prove main results we first show that for every $f \in D_{r}\left(\Omega, \Gamma^{-}\right)$there exists a $u \in D_{r}\left(\Omega, \Gamma^{-}\right)$which solves (BVP). We start with

Lemma 4.1 We can choose a $\lambda_{0} \in \mathbf{R}$ verifying the following properties: Let $\eta \geq 0, s \in \mathbf{R}, \lambda \in \mathbf{C}$ and $u \in C^{1}\left(\bar{\Omega}_{\eta}\right)$ with $u=0$ on $\bar{\Omega} \cap\left\{\phi_{\eta}=0\right\}$. Then we have

$$
\operatorname{Re}\left(\left(L_{(\eta, s)}+\lambda\right) u, u\right)_{\Omega_{\eta}} \geq\left(\operatorname{Re} \lambda-\lambda_{0}\right)\|u\|_{\Omega_{\eta}}^{2}+s\left(u, \phi_{\eta}^{-1} G u\right)_{\Omega_{\eta}} .
$$

Proof. Let us set $Z(x)=\frac{1}{2}\left\{\left(B+B^{*}\right)-\sum_{j=1}^{n}\left(\partial_{j} A_{j}\right)\right\}$. Then it is clear that $Z(x) \gg-\lambda_{0} I$ in $\Omega$ with some $\lambda_{0} \in \mathbf{R}$. Now Green's identity yields

$$
(L u, u)_{\Omega_{\eta}}=\left(u, L^{*} u\right)_{\Omega_{\eta}}+\int_{\partial \Omega_{\eta}}\left\langle A_{\eta b} u, u\right\rangle d \sigma
$$

where $A_{\eta b}$ denotes the boundary matrix in $\Omega_{\eta}$. It follows from $L^{*}=-L+2 Z$ and Lemma 3.3 that

$$
\begin{aligned}
\operatorname{Re}\left(\left(L_{(\eta, s)}+\lambda\right) u, u\right)_{\Omega_{\eta}}= & (u, Z u)_{\Omega_{\eta}}+s\left(u, \phi_{\eta}^{-1} G u\right)_{\Omega_{\eta}} \\
& +\operatorname{Re} \lambda\|u\|_{\Omega_{\eta}}^{2}+\frac{1}{2} \int_{\partial \Omega_{\eta}}\left\langle A_{\eta b} u, u\right\rangle d \sigma .
\end{aligned}
$$

Since the boundary term is non negative, the proof is complete.

Corollary 4.2 There is a $\Lambda \in \mathbf{R}$ such that if $\lambda \in \mathbf{C}$ and $u \in C^{1}(\bar{\Omega})$ with $u \in M$ at $\partial \Omega$ then it follows that

$$
(\operatorname{Re} \lambda-\Lambda)\|u\|_{\Omega} \leq\|(L+\lambda) u\|_{\Omega}
$$

Proof. Lemma 4.1 with $\eta=0, s=0$ gives

$$
\left(\operatorname{Re} \lambda-\lambda_{0}\right)\|u\|_{\Omega}^{2} \leq \operatorname{Re}((L+\lambda) u, u)_{\Omega} \leq\|(L+\lambda) u\|_{\Omega}\|u\|_{\Omega}
$$

which proves the assertion.

Lemma 4.3 For any $r \in \mathbf{Z}_{+}$there is a $\Lambda(r) \in \mathbf{R}$ such that if $u \in$ $D_{r}\left(\Omega, \Gamma^{-}\right),(L+\lambda) u \in H_{r}(\Omega)$ and $\lambda \in \mathbf{C}$ then we have

$$
(\operatorname{Re} \lambda-\Lambda(r))\|u\|_{H_{r}(\Omega)} \leq\|(L+\lambda) u\|_{H_{r}(\Omega)} .
$$

Proof. We proceed by induction on $r$. We first consider the case $r=0$. Using Lemma 3.1 we choose $\left\{u_{\epsilon}\right\} \subset D_{\infty}\left(\Omega, \Gamma^{-}\right)$such that $u_{\epsilon} \rightarrow u,(L+$ 
$\lambda) u_{\epsilon} \rightarrow(L+\lambda) u$ in $L^{2}(\Omega)$. Since we have $u_{\epsilon} \in C^{1}(\bar{\Omega})$ with $u_{\epsilon} \in M$ at $\partial \Omega$, it follows from Corollary 4.2 that

$$
(\operatorname{Re} \lambda-\Lambda)\left\|u_{\epsilon}\right\|_{\Omega} \leq\left\|(L+\lambda) u_{\epsilon}\right\|_{\Omega} .
$$

Letting $\epsilon \downarrow 0$ we get the assertion for $r=0$.

Inductively assume that the statement is true up to $r-1$. Let $u \in$ $D_{r}\left(\Omega, \Gamma^{-}\right)$and $(L+\lambda) u \in H_{r}(\Omega)$. Since $\partial_{k} u \in D_{r-1}\left(\Omega, \Gamma^{-}\right)$and

$$
(L+\lambda) \partial_{k} u=\partial_{k}(L+\lambda) u-\sum_{j=1}^{n}\left(\partial_{k} A_{j}\right) \partial_{j} u-\left(\partial_{k} B\right) u \in H_{r-1}(\Omega),
$$

it follows from the inductive hypothesis that

$$
\begin{aligned}
(\operatorname{Re} \lambda-\Lambda(r-1))\left\|\partial_{k} u\right\|_{H_{r-1}(\Omega)} & \leq\left\|(L+\lambda) \partial_{k} u\right\|_{H_{r-1}(\Omega)} \\
& \leq\left\|\partial_{k}(L+\lambda) u\right\|_{H_{r-1}(\Omega)}+c\|u\|_{H_{r}(\Omega)}
\end{aligned}
$$

with some $c>0$. These estimates prove the assertion for $r$.

Lemma 4.4 We can choose $\eta_{0}>0, c_{0}>0$ and $c_{1}>0$ verifying the following properties: For any $s>1 / 4$ there is a $c(s) \in \mathbf{R}$ such that if $0 \leq \eta \leq \eta_{0}, \operatorname{Re} \lambda>c(s)$ and $u \in C_{0}^{\infty}\left(\Omega_{\eta}\right)$ then we have

$$
\begin{aligned}
(\operatorname{Re} \lambda-c(s))\|u\|_{\Omega_{\eta}}^{2}+ & c_{0}(s-1 / 4)\left\|\phi_{\eta}^{-1 / 2} u\right\|_{\Omega_{\eta}}^{2} \\
& \leq c_{1}\left\|\phi_{\eta}^{1 / 2}\left(L_{(\eta, s)}^{*}+\bar{\lambda}\right) u\right\|_{\Omega_{\eta}}^{2} .
\end{aligned}
$$

Proof. Take a neighborhood $W$ of $\Gamma^{-} \cup \gamma$ as in Lemma 3.4. Then we have $\Omega \cap\left\{\phi_{\eta^{*}}<0\right\} \subset W$ with some $\eta^{*}>0$. Choose $\psi_{i} \in C_{0}^{\infty}\left(\mathbf{R}^{n}\right), i=1,2$ such that

$$
\begin{aligned}
& 0 \leq \psi_{i} \leq 1, \quad \psi_{1}+\psi_{2}=1 \text { in } \Omega, \\
& \operatorname{supp} \psi_{1} \subset W, \quad \psi_{2}=0 \text { in } \Omega \cap\left\{\phi_{\eta^{*}}<0\right\} .
\end{aligned}
$$

From Lemma 4.1 we obtain

$$
\begin{array}{r}
\left(\operatorname{Re} \lambda-\lambda_{0}\right)\|u\|_{\Omega_{\eta}}^{2}+s\left\{\left(\psi_{1} u, \phi_{\eta}^{-1} G u\right)_{\Omega_{\eta}}+\left(\psi_{2} u, \phi_{\eta}^{-1} G u\right)_{\Omega_{\eta}}\right\} \\
\leq \operatorname{Re}\left(\left(L_{(\eta, s)}+\lambda\right) u, u\right)_{\Omega_{\eta}}
\end{array}
$$

It follows from Lemma 3.4 that

$$
\left(\psi_{1} u, \phi_{\eta}^{-1} G u\right)_{\Omega_{\eta}} \geq \delta\left\|\psi_{1}^{1 / 2} \phi_{\eta}^{-1 / 2} u\right\|_{\Omega_{\eta}}^{2}
$$


We turn to $\left(\psi_{2} u, \phi_{\eta}^{-1} G u\right)_{\Omega_{\eta}}$. Since $G$ is bounded in $\Omega$, we get

$$
\left(\psi_{2} u, \phi_{\eta}^{-1} G u\right)_{\Omega_{\eta}} \geq-c_{2}\left\|\psi_{2}^{1 / 2} \phi_{\eta}^{-1 / 2} u\right\|_{\Omega_{\eta}}^{2}
$$

with some $c_{2}>0$. Note that if $x \in \operatorname{supp} \psi_{2} \cap \Omega$ and $0 \leq \eta \leq \eta^{*} / 2$ then we have $\phi_{\eta}(x) \geq \eta^{*} / 2$. Thus it follows that

$$
c_{3}^{-1}\left\|\psi_{2}^{1 / 2} u\right\|_{\Omega_{\eta}} \leq\left\|\psi_{2}^{1 / 2} \phi_{\eta}^{-1 / 2} u\right\|_{\Omega_{\eta}} \leq c_{3}\left\|\psi_{2}^{1 / 2} u\right\|_{\Omega_{\eta}}\left(0 \leq \eta \leq \eta^{*} / 2\right)
$$

with some $c_{3}>0$ which is independent of $u$ and $\eta$ and hence

$$
\left(\psi_{2} u, \phi_{\eta}^{-1} G u\right)_{\Omega_{\eta}} \geq \delta\left\|\psi_{2}^{1 / 2} \phi_{\eta}^{-1 / 2} u\right\|_{\Omega_{\eta}}^{2}-c_{4}\left\|\psi_{2}^{1 / 2} u\right\|_{\Omega_{\eta}}^{2}
$$

with some $c_{4}>0$. Thus the second term of the left-hand side of (4.1) is bounded from below by

$$
\delta\left\|\phi_{\eta}^{-1 / 2} u\right\|_{\Omega_{\eta}}^{2}-c_{4}\|u\|_{\Omega_{\eta}} .
$$

Now noting that

$$
\begin{aligned}
\operatorname{Re}\left(\left(L_{(\eta, s)}+\lambda\right) u, u\right)_{\Omega_{\eta}} & =\operatorname{Re}\left(u,\left(L_{(\eta, s)}^{*}+\bar{\lambda}\right) u\right)_{\Omega_{\eta}} \\
& \leq \frac{1}{4} \delta\left\|\phi_{\eta}^{1 / 2} u\right\|_{\Omega_{\eta}}^{2}+\delta^{-1}\left\|\phi_{\eta}^{1 / 2}\left(L_{(\eta, s)}^{*}+\bar{\lambda}\right) u\right\|_{\Omega_{\eta}}^{2}
\end{aligned}
$$

(4.1) and (4.2) prove the assertion.

Applying the Hahn-Banach theorem we get

Lemma 4.5 We can choose $\eta_{0}>0$ and $\Lambda \in \mathbf{R}$ verifying the following properties: Let $0 \leq \eta \leq \eta_{0}$ and $\operatorname{Re} \lambda>\Lambda$. If $f \in L^{2}\left(\Omega_{\eta}\right)$ then there is a $u \in \phi_{\eta} L^{2}\left(\Omega_{\eta}\right)$ such that $(L+\lambda) u=f$ in $\Omega_{\eta}$.

Proof. Let us set

$$
E=\left\{\phi_{\eta}^{1 / 2}\left(L_{(\eta, 1 / 2)}^{*}+\bar{\lambda}\right) \psi ; \psi \in C_{0}^{\infty}\left(\Omega_{\eta}\right)\right\}
$$

and study the map

$$
T: E \ni \phi_{\eta}^{1 / 2}\left(L_{(\eta, 1 / 2)}^{*}+\bar{\lambda}\right) \psi \mapsto\left(\phi_{\eta}^{-1 / 2} \psi, f\right)_{\Omega_{\eta}} \in \mathbf{C} .
$$

From Lemma 4.4 with $s=1 / 2$ we get

$$
\begin{aligned}
\left|\left(\phi_{\eta}^{-1 / 2} \psi, f\right)_{\Omega_{\eta}}\right|^{2} & \leq\left\|\phi_{\eta}^{-1 / 2} \psi\right\|_{\Omega_{\eta}}^{2}\|f\|_{\Omega_{\eta}}^{2} \\
& \leq 2 c_{0}^{-1} c_{1}\left\|\phi_{\eta}^{1 / 2}\left(L_{(\eta, 1 / 2)}^{*}+\bar{\lambda}\right) \psi\right\|_{\Omega_{\eta}}^{2}\|f\|_{\Omega_{\eta}}^{2}
\end{aligned}
$$


for $\psi \in C_{0}^{\infty}\left(\Omega_{\eta}\right)$. By the Hahn-Banach theorem there is a $w \in L^{2}\left(\Omega_{\eta}\right)$ such that

$$
\left(\phi_{\eta}^{-1 / 2} \psi, f\right)_{\Omega_{\eta}}=\left(\phi_{\eta}^{1 / 2}\left(L_{(\eta, 1 / 2)}^{*}+\bar{\lambda}\right) \psi, w\right)_{\Omega_{\eta}}
$$

for every $\psi \in C_{0}^{\infty}\left(\Omega_{\eta}\right)$. Set $u=\phi_{\eta} w$. Then we have $u \in \phi_{\eta} L^{2}\left(\Omega_{\eta}\right)$ and

$$
\begin{aligned}
\left(\phi_{\eta}^{-1 / 2} \psi, f\right)_{\Omega_{\eta}} & =\left(\phi_{\eta}^{1 / 2}\left(L_{(\eta, 1 / 2)}^{*}+\bar{\lambda}\right) \psi, \phi_{\eta}^{-1} u\right)_{\Omega_{\eta}} \\
& =\left(\psi,\left(L_{(\eta, 1 / 2)}+\lambda\right) \phi_{\eta}^{-1 / 2} u\right)_{\Omega_{\eta}} .
\end{aligned}
$$

Thus we obtain $\left(L_{(\eta, 1 / 2)}+\lambda\right) \phi_{\eta}^{-1 / 2} u=\phi_{\eta}^{-1 / 2} f$ in $\Omega_{\eta}$. Since $L_{(\eta, 1 / 2)} \phi_{\eta}^{-1 / 2}=$ $\phi_{\eta}^{-1 / 2} L$ it follows that $(L+\lambda) u=f$ in $\Omega_{\eta}$ which is the desired assertion.

Proposition 4.6 There is a $\Lambda \in \mathbf{R}$ verifying the following properties: Let $f \in D_{0}\left(\Omega, \Gamma^{-}\right)$and $\operatorname{Re} \lambda>\Lambda$. Then there exists a $u \in D_{0}\left(\Omega, \Gamma^{-}\right)$such that $(L+\lambda) u=f$ in $\Omega$.

Proof. It follows from $f \in D_{0}\left(\Omega, \Gamma^{-}\right)$that $\operatorname{supp} f \subset \bar{\Omega}_{\eta}$ with some $\eta>0$. Using Lemma 4.5 we find $u \in \phi_{\eta} L^{2}\left(\Omega_{\eta}\right)$ satisfying $(L+\lambda) u=f$ in $\Omega_{\eta}$. Let $u^{0}$ be $u$ in $\Omega_{\eta}$ and zero elsewhere. We prove that $(L+\lambda) u^{0}=f$ in $\Omega$ that shows the assertion since it is clear that $u^{0} \in D_{0}\left(\Omega, \Gamma^{-}\right)$. To do so it is sufficient to show that

$$
\left(u^{0},\left(L^{*}+\bar{\lambda}\right) \psi\right)_{\Omega}-(f, \psi)_{\Omega}=0
$$

for every $\psi \in C_{0}^{\infty}(\Omega)$. Choose $\chi(t) \in C_{0}^{\infty}(\mathbf{R})$ so that supp $\chi \subset(-1,1)$ and $\chi=1$ near $t=0$ and set $\chi_{\epsilon}(t)=\chi\left(\epsilon^{-1} t\right)$ for $\epsilon>0$. Let $\psi \in C_{0}^{\infty}(\Omega)$. Then we get

$$
\begin{aligned}
\left(u^{0},\left(L^{*}+\bar{\lambda}\right) \psi\right)_{\Omega}-(f, \psi)_{\Omega}= & \left(u,\left(L^{*}+\bar{\lambda}\right) \psi\right)_{\Omega_{\eta}}-(f, \psi)_{\Omega_{\eta}} \\
= & \left(u,\left(L^{*}+\bar{\lambda}\right)\left(1-\chi_{\epsilon}\left(\phi_{\eta}\right)\right) \psi\right)_{\Omega_{\eta}} \\
& -(f, \psi)_{\Omega_{\eta}}+\left(u,\left(L^{*}+\bar{\lambda}\right) \chi_{\epsilon}\left(\phi_{\eta}\right) \psi\right)_{\Omega_{\eta}}
\end{aligned}
$$

Since $\left(1-\chi_{\epsilon}\left(\phi_{\eta}\right)\right) \psi \in C_{0}^{\infty}\left(\Omega_{\eta}\right)$, the right-hand side of (4.3) is

$$
\begin{aligned}
& \left(f,\left(1-\chi_{\epsilon}\left(\phi_{\eta}\right)\right) \psi\right)_{\Omega_{\eta}}-(f, \psi)_{\Omega_{\eta}}+\left(u,\left(L^{*}+\bar{\lambda}\right) \chi_{\epsilon}\left(\phi_{\eta}\right) \psi\right)_{\Omega_{\eta}} \\
& \left.=-\left(f, \chi_{\epsilon}\left(\phi_{\eta}\right)\right) \psi\right)_{\Omega_{\eta}}+\left(u, \chi_{\epsilon}\left(\phi_{\eta}\right)\left(L^{*}+\bar{\lambda}\right) \psi\right)_{\Omega_{\eta}} \\
& \quad-\sum_{j=1}^{n}\left(u, \partial_{j}\left(\chi_{\epsilon}\left(\phi_{\eta}\right)\right) A_{j} \psi\right)_{\Omega_{\eta}}
\end{aligned}
$$




$$
=I_{1}+I_{2}+I_{3}
$$

The Dominated convergence theorem shows that $I_{1}$ and $I_{2}$ go to zero when $\epsilon \downarrow 0$. We turn to $I_{3}$. Note that

$$
\begin{aligned}
I_{3} & =-\sum_{j=1}^{n}\left(u, \epsilon^{-1} \chi^{\prime}\left(\epsilon^{-1} \phi_{\eta}\right)\left(\partial_{j} \phi\right) A_{j} \psi\right)_{\Omega_{\eta}} \\
& =-\left(u, \epsilon^{-1} \chi^{\prime}\left(\epsilon^{-1} \phi_{\eta}\right) G \psi\right)_{\Omega_{\eta}} .
\end{aligned}
$$

Since $u=\phi_{\eta} w$ with some $w \in L^{2}\left(\Omega_{\eta}\right)$, it follows that

$$
I_{3}=-\left(w, \epsilon^{-1} \phi_{\eta} \chi^{\prime}\left(\epsilon^{-1} \phi_{\eta}\right) G \psi\right)_{\Omega_{\eta}} .
$$

Noticing that $\left|t \chi^{\prime}(t)\right| \leq c$ with some $c>0$ and $G$ is bounded in $\Omega$ the Dominated convergence theorem again proves that $I_{3} \rightarrow 0$ as $\epsilon \downarrow 0$. This completes the proof.

Proposition 4.7 For any $r \in \mathbf{Z}_{+}$there is a $\Lambda(r)$ verifying the following properties: Let $u \in D_{0}\left(\Omega, \Gamma^{-}\right), f \in D_{r}\left(\Omega, \Gamma^{-}\right), \operatorname{Re} \lambda>\Lambda(r)$ and assume that $(L+\lambda) u=f$ in $\Omega$. Then we have $u \in D_{r}\left(\Omega, \Gamma^{-}\right)$.

Proof. Let $u \in D_{0}\left(\Omega, \Gamma^{-}\right)$. Then we have supp $u \subset \Omega_{\eta_{0}}$ with some $\eta_{0}>0$. Note that $\bar{\Omega}_{\eta_{0}} \cap\left(\Gamma^{-} \cup \gamma\right)=\emptyset$. Thus we can repeat the same reasoning as in Tartakoff [11] to conclude the assertion.

We now give the proofs of Theorem 2.2 and Proposition 2.3.

Proof of Theorem 2.2. We first suppose that $f \in D_{r}\left(\Omega, \Gamma^{-}\right)$. By Proposition 4.6 there is a $u \in D_{0}\left(\Omega, \Gamma^{-}\right)$satisfying $(L+\lambda) u=f$ in $\Omega$. It follows from Proposition 4.7 that $u \in D_{r}\left(\Omega, \Gamma^{-}\right)$, in particular, $u \in X_{r}\left(\Omega, \Gamma^{-}\right)$. In view of Lemma 3.2 $u$ is a weak solution to (BVP). Moreover by Lemma 4.3 we get

$$
(\operatorname{Re} \lambda-\Lambda(r))\|u\|_{H_{r}(\Omega)} \leq\|(L+\lambda) u\|_{H_{r}(\Omega)}=\|f\|_{H_{r}(\Omega)} .
$$

Let $f \in X_{r}\left(\Omega, \Gamma^{-}\right)$. Since $D_{r}\left(\Omega, \Gamma^{-}\right)$is dense in $X_{r}\left(\Omega, \Gamma^{-}\right)$, the assertion can be proved by standard limiting arguments.

Proof of Proposition 2.3. Assuming that $u \in L^{2}(\Omega)$ is a weak solution to (BVP) with $f=0$ we show $u=0$. Let $g \in D_{0}\left(\Omega, \Gamma^{+}\right)$. Repeating the same arguments in Proposition 4.6 we can find $v \in D_{0}\left(\Omega, \Gamma^{+}\right)$such that $\left(L^{*}+\bar{\lambda}\right) v=g$ in $\Omega$. We choose $\left\{v_{\epsilon}\right\} \subset D_{\infty}\left(\Omega, \Gamma^{+}\right)$satisfying $v_{\epsilon} \rightarrow$ 
$v,\left(L^{*}+\bar{\lambda}\right) v_{\epsilon} \rightarrow g$ in $L^{2}(\Omega)$. Note that $v_{\epsilon} \in M^{*}$ at $\partial \Omega$ and hence

$$
\left(u,\left(L^{*}+\bar{\lambda}\right) v_{\epsilon}\right)_{\Omega}=0 .
$$

Letting $\epsilon \downarrow 0$ we get $(u, g)_{\Omega}=0$. Since $D_{0}\left(\Omega, \Gamma^{+}\right)$is dense in $L^{2}(\Omega)$, we conclude that $u=0$ and hence the assertion.

\section{Initial boundary value problem}

Let $\Omega, L$ and $M$ be as described in the preceding sections. We consider the following initial boundary value problem

$$
\text { (IBVP) }\left\{\begin{aligned}
\partial_{t} u+L u=f(t, x) & \text { in } \quad(0, \infty) \times \Omega \\
u(0, x)=u_{0}(x) & \text { in } \Omega \\
u(t, x) \in M(x) & \text { for } \quad(t, x) \in[0, \infty) \times \partial \Omega .
\end{aligned}\right.
$$

To solve this initial boundary value problem (IBVP) we apply the HilleYosida theorem. Let us regard $L$ as a linear operator $L: X_{r}\left(\Omega, \Gamma^{-}\right) \rightarrow$ $X_{r}\left(\Omega, \Gamma^{-}\right)$with domain

$$
\begin{aligned}
& \mathcal{D}(L)=\left\{u \in X_{r}\left(\Omega, \Gamma^{-}\right) ; L u \in X_{r}\left(\Omega, \Gamma^{-}\right)\right. \text {and } \\
& \left.\quad\left(u, L^{*} \psi\right)_{\Omega}=(L u, \psi)_{\Omega} \text { for every } \psi \in C^{\infty}(\bar{\Omega}) \text { with } \psi \in M^{*} \text { at } \partial \Omega\right\} .
\end{aligned}
$$

We write simply $X_{r}$ for $X_{r}\left(\Omega, \Gamma^{-}\right)$.

Proposition 5.1 Let $r \in \mathbf{Z}_{+}$. Then $-L$ generates a $C_{0}$ semi-group.

For the proof we note that

Lemma 5.2 Let $u \in X_{r}$ and $f \in X_{r}$. In order that $u \in \mathcal{D}(L)$ and $(L+$ $\lambda) u=f$ it is necessary and sufficient that $u$ is a weak solution to (BVP).

Proof. Since

$$
\left(u,\left(L^{*}+\bar{\lambda}\right) \psi\right)_{\Omega}-(f, \psi)_{\Omega}=\left(u, L^{*} \psi\right)_{\Omega}-(L u, \psi)_{\Omega}
$$

for every $\psi \in C^{\infty}(\bar{\Omega})$ with $\psi \in M^{*}$ at $\partial \Omega$ and hence the assertion.

Proof of Proposition 5.1. In view of the Hille-Yosida theorem (see [10] for example), it is enough to check the following two conditions:

(1) $L$ is a closed operator with domain $\mathcal{D}(L)$ dense in $X_{r}$.

(2) There is a $\Lambda(r) \in \mathbf{R}$ such that if $\operatorname{Re} \lambda>\Lambda(r)$ then we have $-\lambda \in \rho(L)$ 
and

$$
(\operatorname{Re} \lambda-\Lambda(r))\left\|(L+\lambda)^{-1}\right\| \leq 1
$$

where $\rho(L)$ denotes the resolvent set of $L$.

Since $D_{\infty}\left(\Omega, \Gamma^{-}\right) \subset \mathcal{D}(L)$ then $\mathcal{D}(L)$ is dense in $X_{r}$. Now let $\left\{u_{n}\right\} \subset$ $\mathcal{D}(L), u \in X_{r}, f \in X_{r}$ and assume that $u_{n} \rightarrow u, L u_{n} \rightarrow f$ in $H_{r}(\Omega)$. Since $u_{n} \in \mathcal{D}(L)$, it follows from Lemma 5.2 that $u_{n}$ is a weak solution to (BVP) with the right-hand side $f_{n}=L u_{n}$ and $\lambda=0$. It is clear that $u$ is a weak solution to (BVP) and hence, in view of Lemma 5.2, we conclude that $u \in \mathcal{D}(L)$ and $L u=f$ which proves (i).

We turn to the assertion (ii). Assume that $v \in \mathcal{D}(L)$ verifies $(L+$ $\lambda) v=0$. From Lemma 5.2 $v$ is a weak solution to (BVP) with $f=0$. By Proposition 2.3 we have $v=0$. This proves the injectivity of the mapping $(L+\lambda): \mathcal{D}(L) \rightarrow X_{r}$. Let $f \in X_{r}$. From Theorem 2.2 there exists a weak solution $u \in X_{r}$ to (BVP) such that

$$
(\operatorname{Re} \lambda-\Lambda(r))\|u\|_{H_{r}(\Omega)} \leq\|f\|_{H_{r}(\Omega)} .
$$

From Lemma 5.2 it follows that $u \in \mathcal{D}(L)$ and $(L+\lambda) u=f$. Hence the mapping $(L+\lambda)$ is surjective. The desired estimate follows from (5.1).

Let $\{T(t)\}_{t \geq 0}$ be a $C_{0}$ semi-group with generator $-L$ obtained by Proposition 5.1. Then we have

Proposition 5.2 Let $r \in \mathbf{Z}_{+}$and $T>0$. Assume that $u_{0} \in \mathcal{D}(L), f \in$ $C^{0}\left([0, T] ; X_{r}\right), f(t) \in \mathcal{D}(L)(0 \leq t \leq T)$ and $L f \in C^{0}\left([0, T] ; X_{r}\right)$. Then (IBVP) has a unique solution $u \in C^{1}\left([0, T] ; X_{r}\right)$ which is given by

$$
u(t)=T(t) u_{0}+\int_{0}^{t} T(t-s) f(s) d s \quad(0 \leq t \leq T) .
$$

Corollary 5.1 Let $r \in \mathbf{Z}_{+}$and $T>0$. Assume that $u_{0} \in X_{r+1}, f \in$ $C^{0}\left([0, T] ; X_{r+1}\right)$. Then (IBVP) has a unique solution $u \in C^{1}\left([0, T] ; X_{r}\right)$ which is given by (5.2). Furthermore, $u$ satisfies

$$
\begin{aligned}
\|u(t)\|_{H_{r}(\Omega)} \leq & e^{\Lambda(r) t}\left\|u_{0}\right\|_{H_{r}(\Omega)} \\
& +\int_{0}^{t} e^{\Lambda(r)(t-s)}\|f(s)\|_{H_{r}(\Omega)} d s \quad(0 \leq t \leq T)
\end{aligned}
$$

where $\Lambda(r) \in \mathbf{R}$ is independent of $u_{0}, f$ and $T$. 
Proof. Since $X_{r+1} \subset \mathcal{D}(L)$ then the first part is clear. To get the estimate (5.3) it suffices to note that $\|T(t)\| \leq e^{\Lambda(r) t}(t \geq 0)$ follows from the HilleYosida theorem and the proof of Proposition 5.1.

Theorem 5.5 Let $r \in \mathbf{Z}_{+}, r \geq 1$. Assume that $u_{0} \in X_{r}, f \in C^{0}([0, \infty)$; $\left.X_{r}\right)$. Then there is a unique solution $u \in C^{0}\left([0, \infty) ; X_{r}\right) \cap C^{1}\left([0, \infty) ; X_{r-1}\right)$ to the initial boundary value problem (IBVP).

Proof. $\quad$ Fix $T>0$. Since $X_{r+1}, C^{0}\left([0, T] ; X_{r+1}\right)$ are dense in $X_{r}, C^{0}([0, T]$; $\left.X_{r}\right)$ respectively, we can choose $\left\{u_{0 n}\right\} \subset X_{r+1}$ and $\left\{f_{n}\right\} \subset C^{0}\left([0, T] ; X_{r+1}\right)$ so that

$$
u_{0 n} \rightarrow u_{0} \quad \text { in } \quad H_{r}(\Omega), \quad f_{n} \rightarrow f \quad \text { in } \quad C^{0}\left([0, T] ; X_{r}\right) .
$$

By Corollary 5.4 there is a solution $u_{n} \in C^{1}\left([0, T] ; X_{r}\right)$ to (IBVP) replaced $u_{0}, f$ by $u_{0 n}, f_{n}$. Applying the estimate (5.3) to $u_{n}-u_{m}$ we find that $\left\{u_{n}\right\}$ is a Cauchy sequence in $C^{0}\left([0, T] ; X_{r}\right)$. Let $u \in C^{0}\left([0, T] ; X_{r}\right)$ be the limit of $\left\{u_{n}\right\}$. Now $u_{n}$ satisfies

$$
u_{n}(t)=u_{0 n}+\int_{0}^{t}\left\{-\left(L u_{n}\right)(s)+f_{n}(s)\right\} d s \quad(0 \leq t \leq T) .
$$

Letting $n \rightarrow \infty$ we obtain

$$
u(t)=u_{0}+\int_{0}^{t}\{-(L u)(s)+f(s)\} d s \quad(0 \leq t \leq T)
$$

which shows that $u \in C^{0}\left([0, T] ; X_{r}\right) \cap C^{1}\left([0, T] ; X_{r-1}\right)$ is a solution to (IBVP). The uniqueness follows from Corollary 5.4. Since $T>0$ is arbitrary we get the desired assertion.

\section{References}

[1] Friedrichs K.O., The identity of weak and strong extensions of differential operators. Trans. Amer. Math. Soc. 55 (1944), 132-151.

[2] Friedrichs K.O., Symmetric positive linear differential opreators. Comm. Pure Appl. Math. 11 (1958), 333-418.

[ 3 ] Hörmander L., Linear Partial Differential Operators. Springer-Verlag, Berlin-Göttingen-Heidelberg, 1963.

[4] Lax P.D. and Phillips R.S., Local boundary conditions for dissipative symmetric linear differential operators. Comm. Pure Appl. Math. 13 (1960), 427-455.

[5] Moyer R., On the nonidentity of weak and strong extensions of differential operators. Proc. Amer. Math. Soc. 19 (1968), 487-488. 
[6] Osher S., An ill-posed problem for a hyperbolic equation near a corner. Bull. Amer. Math. Soc. 79 (1973), 1043-1044.

[7] Phillips R.S. and Sarason L., Singular symmetric positive first order differential operators. J. Math. Mech. 15 (1966), 235-272.

[8] Rauch J., Symmetric positive systems with boundary characteristic of constant multiplicity. Trans. Amer. Math. Soc. 291 (1985), 167-187.

[9] Rauch J., Boundary value problem with nonuniformly characteristic boundary. J. Math. Pure et Appl. 73 (1994), 347-353.

[10] Tanabe H., Functional Analysis I (in Japanese), Jikkyo Shyuppan, Tokyo, 1978.

[11] Tartakoff D., Regularity of solutions to boundary value problems for first order systems. Indiana Univ. Math. J. 21 (1972), 1113-1129.

Tatsuo Nishitani

Department of Mathematics

Osaka University

Machikaneyama 1-16

Toyonaka Osaka 560, Japan

E-mail: tatsuo@math.wani.osaka-u.ac.jp

Masahiro Takayama

Department of Mathematics

Osaka University

Machikaneyama 1-16

Toyonaka Osaka 560, Japan 